\title{
Blob adaptation through frames analysis for dynamic fire detection
}

\author{
Indrabayu', Rahmat Hardian Putra ${ }^{2}$, Ingrid Nurtanio ${ }^{3}$, Intan Sari Areni ${ }^{4}$, Anugrayani Bustamin ${ }^{5}$ \\ 1,2,3,5 Department of Informatics Engineering, Hasanuddin University, Indonesia \\ ${ }^{4}$ Department of Electrical Engineering, Hasanuddin University, Indonesia
}

\begin{abstract}
Article Info
Article history:

Received Jan 17, 2020

Revised Mar 31, 2020

Accepted May 2, 2020

Keywords:

Blob analysis

Computer vision

Distance estimation

Fire detection

Pinhole model

ABSTRACT

This study was aiming at helping visually impaired people to detect and estimate the fire distance. Blind people had difficulty knowing the existence of fire at a safe distance; hence the possibility of burning could occur. The color models and blob analysis methods were used to detect the presence of fire in the blind path. Before the fire detection stage, the cascade of the HSV and RGB color models was applied to segment the reddish fire color. The size and shape of a dynamic fire were the parameters used in this paper to distinguish fire from non-fire objects. Changes in the area of the fire object obtained at the Blob analysis stage per 10 frames were the main contributions and novelty in this paper. After the fire is detected, the calculation of the fire distance to a blind person was completed using a pinhole model. This research used 35 data videos with a resolution of 480x640 pixels. The results showed that the fire detection system and the distance estimation achieved an accuracy of $88.86 \%$ and the MSE of 0.0358 , respectively.
\end{abstract}

This is an open access article under the CC BY-SA license.

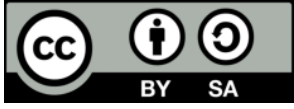

\section{Corresponding Author:}

Indrabayu,

Department of Informatics Engineering,

Hasanuddin University,

J1. Poros Malino KM. 6, Bontomarannu, Gowa, South Sulawesi 92172, Indonesia.

Email: indrabayu@unhas.ac.idw

\section{INTRODUCTION}

Blindness is a condition where a person experiences vision impairment to a certain degree depending upon many factors, such as illness, accident, or other cause. Blind people face many challenges, such as difficulty to interact even in daily activities. Activities performed by blind people can endanger their safety, particularly activities involving sharp objects and objects that can cause fires. Thus, blind people tend to need the help of others [1]. According to FEMA (Federal Emergency Management Agency), blind people have difficulties to feel the presence of fire at a safe distance, increasing the chance of being injured. Blind people find it hard to recognize the early signs of a fire. Therefore, even small fires can become a threat to the blind [1].

A new concept of smart sticks had been proposed to help blind people detect obstacles and even help their navigation using GPS. However, this smart stick is still relatively expensive [2]. Another research proposed a smart stick integrated with the ultrasonic, light, and water sensor [3-5]. Other various research also has been conducted to offer solutions to help blind people but rarely able to recognize fire as a danger. Recently, computer vision technology has also been implemented in applications and systems and intended to assist blind people. It has been used in a wide range of fields, especially in face recognition. Several previous research has proven that computer vision technology is reliable and accurate in recognizing the human 
face [6-9]. Computer vision has shown notable results in recognizing non-solid objects as well. For instance, research implemented computer vision to detect fire and smoke using color features and color models. The block method is adopted to reduce error detection or false alarm caused by other objects because this method can recognize natural movement features of fire. From the predetermined scenario, this system produces $100 \%$ accuracy without the occurrence of false alarms [10].

Segmentation is one important process in image processing technology. Jaiswal et al. introduced a new method of image segmentation by minimizing the previous 3-dimensional segmentation into 2-dimensional only with genetic algorithms. The results obtained show clear segments between objects based on their color features [11]. Another segmentation process was also tested on a noisy eye image. The use of contour-based feature segmentation, including brightness, color, and texture, is the focus of this study. From these results, the CNN algorithm is used to cluster parts of the eye that will be further processed, such as iris, pupils, and sclera [12]. Research conducted by Mengistu also compares the performance of several segmentation techniques. The object of this research is Ethiopian coffee varieties. Otsu, fuzzy c-means (FCM), and K-means methods as segmentation methods have been tested. The results of the segmentation are then classified with backpropagation neural network (BPNN). The best performance was obtained at $94.54 \%$ using FCM as a segmentation method [13].

Moreover, Yadav et al. proposed a fire detection system using videos taken with ordinary cameras. This study combines several methods to detect fire, such as the color detection method using the RGB color model, motion detection method to detect the fire movement, and the gray cycle detection method to detect gray color cycles of smoke produced by the fire. Also, the area dispersion detection method is applied to detect the spreading of fire pixels from several frames. This new system simulates an existing fire detection technique by adding optimization to reduce false alarms and improve accuracy. The percentage of system performance offered is $92.31 \%$, with a false alarm rate of $7.69 \%$ [14].

Research on distance estimation has also been conducted. Sadreddini et al. examined a distance calculation method using a single camera for indoor environments. In this method, the initial step is to extract the appropriate vertical floor line from the snapshots sequence. Moreover, the line passing through the base side of the object is found simultaneously with the floor line. Then, the part of the floor line starting from this point is measured in pixels, by taking the intersection point of these lines, and converted to distance between the camera and the object. This method can be applied to buildings with regular lines on the floor. The results of this study showed that the proposed method had a good ability to measure distances, but the greater the distance to be measured, the error between the distance calculated and the actual distance will also be greater. However, at a certain distance, the error will be constant [15].

Another method for distance estimation was proposed to estimate the distance between the colored object to the camera using image processing. First, image color filtering based on the HSV color model was performed to the image. Then, object detection was applied to label eight nearest connected components with the same value using the Moore-neighborhood tracing algorithm. The distance of the object was estimated using the single point projection principle of the object height in the image, represented by the bounding rectangle, and the actual object's height. Finally, an experiment was conducted using objects with different colors and distance variation in centimeters. The results showed that the method could estimate the real distance of the colored object from the camera [16].

Furthermore, research conducted by Rahman et al. used a new method to calculate the distance from an object with only a single image. The correlation between real distance and object height in the image is used in the training process so that the correlation between the two parameters is obtained. These results are used to estimate the distance of the real object through the height of pixels in the image. The philosophy of this method comes from seafarers. They use this method when they need to determine their distance from the coastline using lighthouse projection. This proposed system reached an accuracy of 98.76\% [17].

Abdurrasyid et al. [18] have researched barrier objects in blind people such as stairs, doors, and walls. The resulting output in the form of a warning in the form of sound data so that people with visual impairments can avoid objects obstructions during walking. Different from previous research, vehicle detection systems were also developed using the OTSU method and K-means clustering in 2019 and the merging of the R-CNN and Kalman Filter methods in 2020 [19-20]. The otsu method is also still used for detection of human movement objects by Soeleman et al. by utilizing the adaptive threshold in the gaussian mixture algorithm [21].

This research proposes an Android-based system for detecting fire and measuring the distance to help blind people avoid the danger of being exposed to fire. Blob analysis and color models are used to detect fire based on the reddish color of the fire. However, many other objects can be detected as fire because the colors resemble fire, such as street lights or vehicle lights. Therefore, the dynamic motion of fire is used to distinguish fire from the objects around it. To estimate the relative distance of the blind and fire used Pinhole Model. Similar research has been conducted in dealing with fire dynamics. However, the researches only consider fire as a dynamic object while in this paper subject (camera) is also moving considerably. 


\section{RESEARCH METHOD}

This research comprises five main stages, which are data acquisition, image segmentation, image enhancement, fire detection, and distance estimation, as shown in Figure 1.

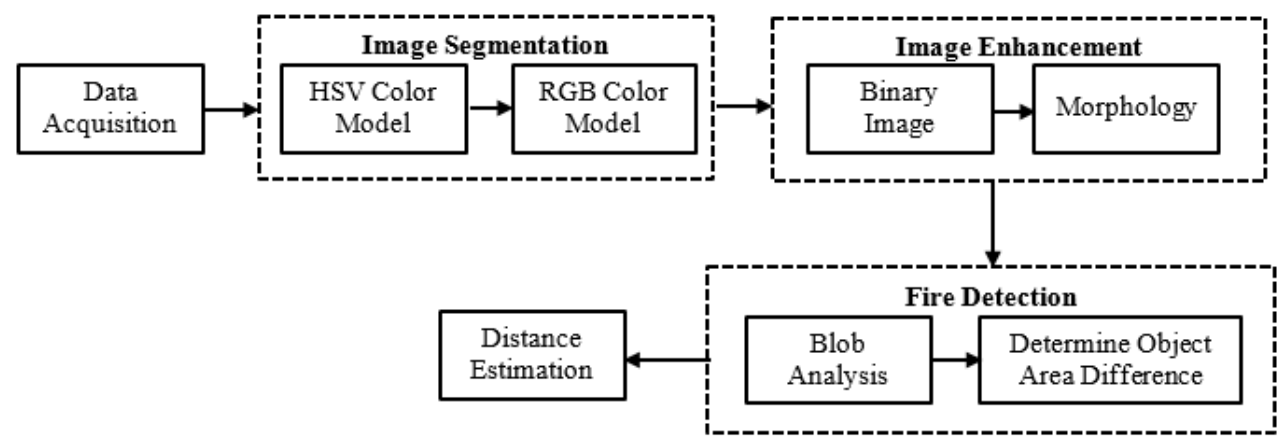

Figure 1. Fire detection system design

\subsection{Data acquisition}

In the data acquisition process, a point of view camera (Povie) is employed as the smartphone holder that keeps the camera in a stable and safe position for blind people. Povie is placed on the neck of the blind. Illustration of the data collection process using povie camera can be seen in Figure 2. The input data are video footage of fire, taken with 1920x1080 resolutions smartphone camera under the scenario of use by the blind. The video is recorded at 30 frames per second. The data are taken directly by recording fire at day and night conditions and in different places such as home yards, roadside, and open fields. The system will process the input video by reading each frame of the video sequentially. The frames have a resolution of $1920 \times 1080$ with landscape orientation. These frames need to be preprocessed to improve the performance of the system. The preprocessing can be done by cropping the video with a ratio of 3:4 to change the resolution into $810 \times 1080$, then cropping the video with a factor scale of 0.592592 . This process will modify the frames into portrait images with $480 \times 640$ resolution. This setting is intended to lower system computing time and to narrow down the field of view because the obstacles to be avoided are on the track of the blind.

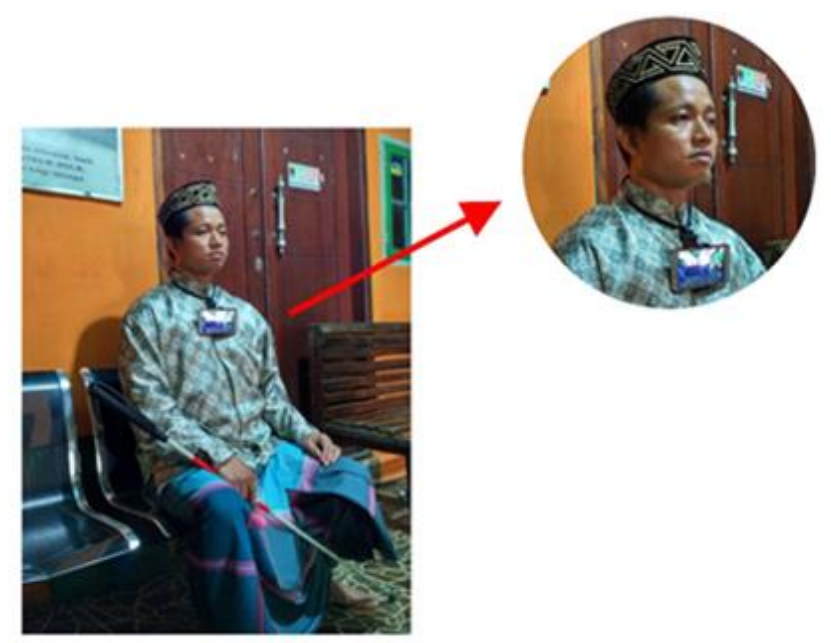

Figure 2. Data collection process using povie

\subsection{Image segmentation}

This research implements the image segmentation method, which is a process for identifying and distinguishing foreground from background content. Segmentation is used to partition an image into homogeneous regions concerning some image features. Usually, the output of image segmentation is a binary 
image where the desired foreground is white (equal to 1), and the undesired background is black (equal to 0 ). Segmentation can also divide images into several parts based on the characteristics of the pixels. Commonly used image segmentation methods are thresholding, active contour, color segmentation, edge detection, watershed, and Hough transformation. Color segmentation uses a color model to segment objects from images. The most common color models are RGB and HSV. In this study, the segmentation phase is carried out with a two-stage cascade color model, i.e. HSV and RGB color models. The RGB color model is an additive color model, which red, green and blue light are mixed to reproduce a broad range of colors. The intensity of each color can vary from 0 to 255 , which gives $16,777,216$ different colors. To produce a new color, three colored light rays must be combined. Without intensity, each of the three colors is perceived as black, while full intensity displays white color. This color space describes colors in terms of their shade and their brightness value.

The HSV color model defines colors in terms of hue, saturation, and value. Hue expresses true colors, such as red, violet, and yellow. Hue is associated with the wavelength of light. Saturation defines the purity of a color, which indicates how much white is given to the color. Value is an attribute that declares the amount of light received by the eye regardless of the color [22]. In the HSV segmentation stage, this research particularly uses only pixels that have a reddish color, which represents the color of fire. In HSV color model, fire color are within 0-0.9792 Hue, 0-0.6473 Saturation and 0.8118-1 Value. The color segmentation process is shown in Figure 3. Figure 3(a) displays the input fire image, and Figure 3(b) shows the results of segmentation with the HSV model. Next, the pixel filtering process is carried out in Figure 3(b) based on a predetermined range of HSV values. So that only the color of the flame will be obtained, as shown in Figure 3(c). However, there are still some objects that have a color similar to fire and fall into the range used, so that the second segmentation using the RGB color model is used to reduce this segmentation error. Figure 3(d) shows the results of the RGB color model segmentation.

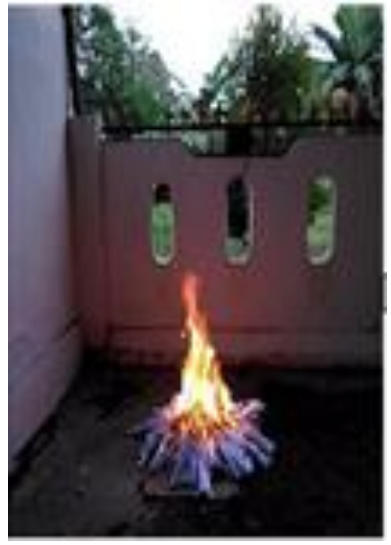

(a)

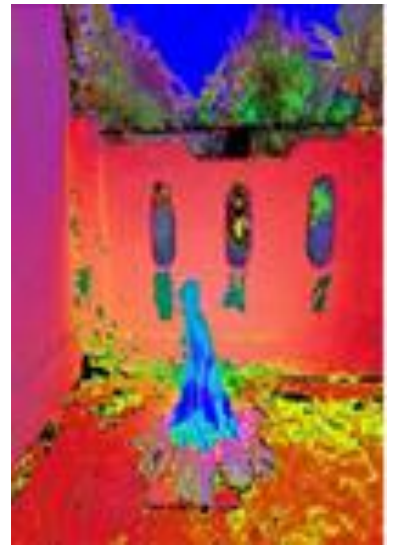

(b)

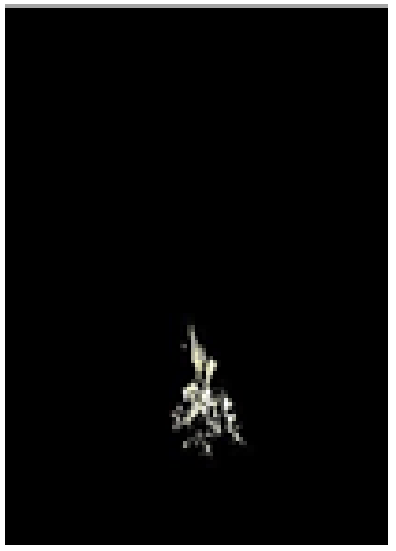

(c)

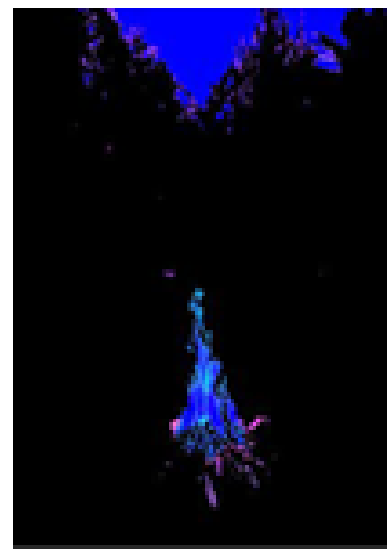

(d)

Figure 3. Color segmentation process, (a) The input fire image example, (b) HSV color model result,

(c) The pixel filtering result, (d) RGB color model result

\subsection{The proposed algorithm for fire detection}

Fire constantly experiences size change and irregular movement. Due to this characteristic, blob analysis is implemented to analyze the change of the segmented object. Blob stands for a binary large object. The main advantages of this technique are its high flexibility and excellent performance. Its limitations are background-foreground relation requirement and pixel precision. The purpose of blob extraction is to locate the blob, which is the connected region corresponding to the objects being inspected in a binary image. A blob consists of a connected pixel group. In determining the blob value, several things must be known to produce optimal blob. The determination of a blob width on each object in the foreground segmentation process needs to be analyzed because the blob value for each object will be different. This is influenced by object features such as size, type, and video data retrieval techniques. The process starts from marking the foreground area which is considered an object, then collecting the data of the segmented area, such as the position of the initial pixel, the length of the $x$-axis and the $y$-axis, and the area of pixels [23]. The proposed algorithm for fire detection is shown in the pseudocode: 


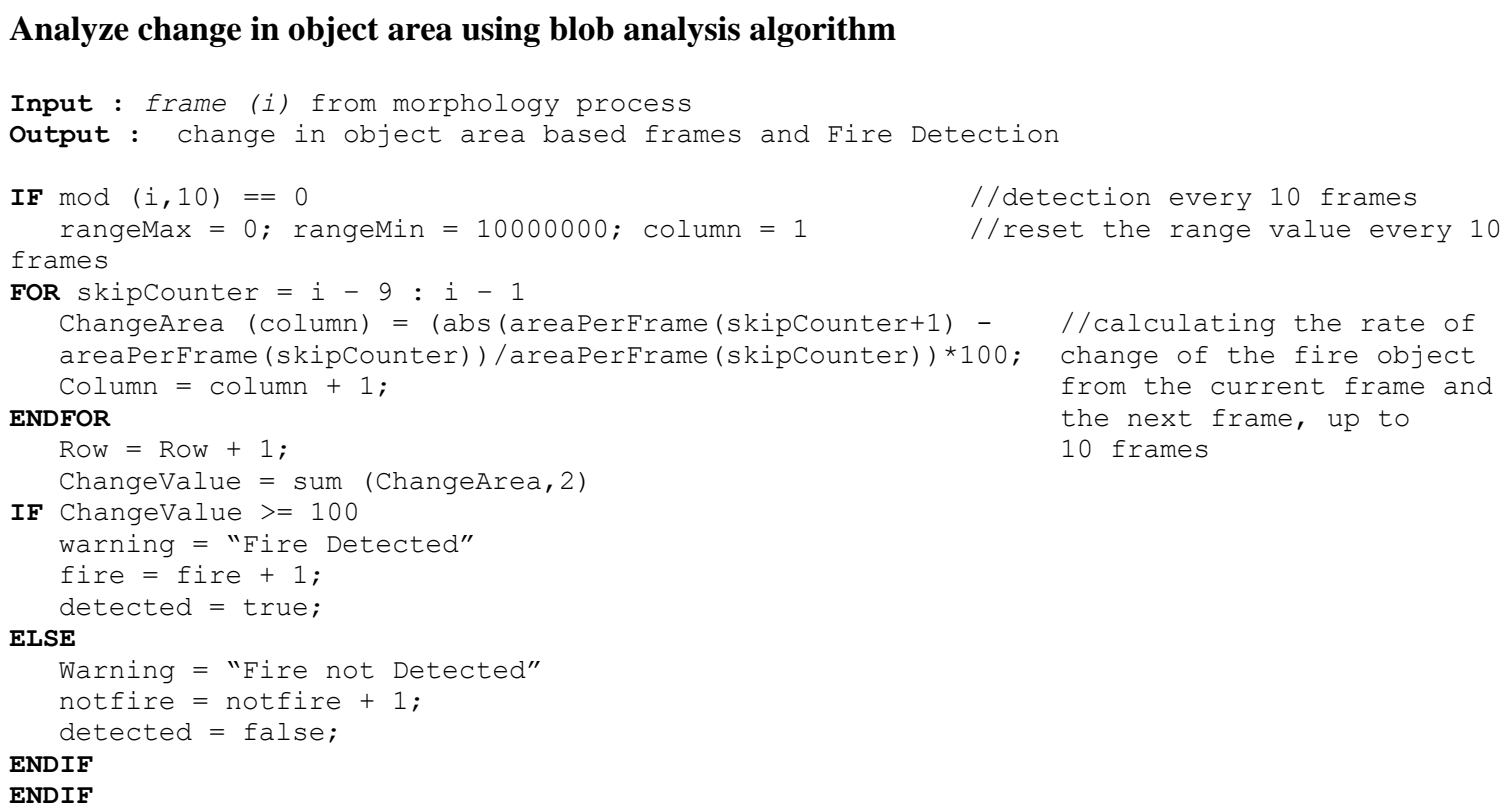

The threshold value is determined based on the experiment using positive and negative data. Positive data are videos of actual fire and negative data are a video containing objects that have a similar color to fire. From the experiments, the positive data have an average change of size above 100, while the negative data video has an average size change below 100. So the used threshold value for detecting fire in this paper is 100 .

\subsection{Distance}

After the fire has been detected, the distance estimation is calculated using the pinhole model. Pinhole model or pinhole camera is a method of taking pictures based on geometric projection between 3D points and 2D points that correspond to projections in the image. The Pinhole camera can project real-world images to a light-tight box or room with an aperture in the middle on one side of the box. The principle of pinhole cameras is simply described as follows. Objects from the landscape reflect light in all directions. The small size of the box hole only allows a small amount of light to pass and project the object upside down. The geometric mapping model of $3 \mathrm{D}$ space into $2 \mathrm{D}$ space is called perspective projection. The midpoint of perspective projection is the intersection of the optical/camera center and the line perpendicular to the image passing through the optical center also called the optical axis. While the point of image intersection with the optical axis called the main point [24], the illustration of the pinhole model can be seen in Figure 4 [25].

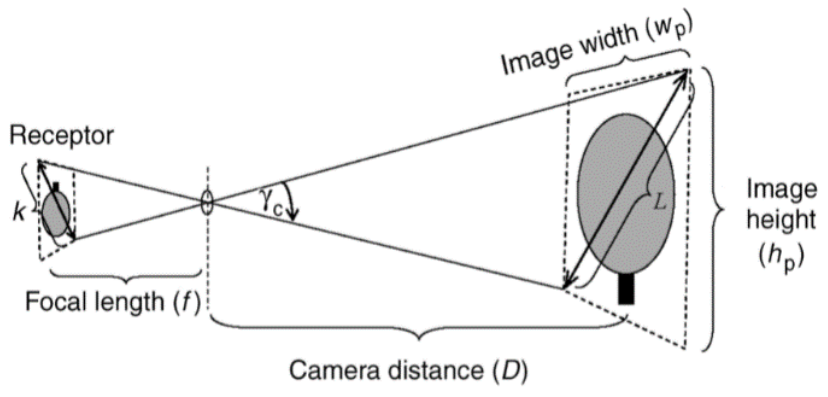

Figure 4. Pinhole model

Figure 4 shows the projection of a 3D object from the real world into a 2D plane through a hole. The diagonal length of the projected image $(k)$ can be calculated easily with only information on the focal length (f) and the diagonal length of the object $(L)$ and the object distance from the camera $(D)$. The view angle $\left(\gamma_{c}\right)$ of the image is defined as the angle made by the diagonal of the picture. The pinhole model searches for the closest object to the camera by calculating the $Y$-axis coordinate value of the object. Then, 
the distance is calculated by comparing the coordinate object value in pixels and the reference value, which is a point in real-world that is used as the reference to find the estimated distance. The pixel references $\left(d_{\text {ref }}\right)$ and actual distance references $\left(p_{\text {ref }}\right)$ can be seen in Table 1 .

Table 1. Actual distance and pixel references

\begin{tabular}{cc}
\hline$d_{\text {ref }}(\mathrm{m})$ & $p_{\text {ref }}(\mathrm{px})$ \\
\hline 1 & 640 \\
2 & 618 \\
3 & 505 \\
4 & 439 \\
5 & 400 \\
6 & 374 \\
7 & 354 \\
8 & 339 \\
\hline
\end{tabular}

Based on Table 1, an object with pixel distance $(p)$ of 620 px from the $Y$-axis will use 618 px as the nearest pixel reference and $2 \mathrm{~m}$ as the nearest actual distance reference. The actual distance $(d)$ of the object is given by (1). The illustration of actual and image (pixel) distance comparison shown in Figure 5. The result of the fire detection system is determined based on the total change in object size. If it exceeds the predetermined threshold, the object will be detected as fire; otherwise, it will not be detected as fire. For objects that are positively detected as fire, a bounding box will appear and the estimated distance information will be displayed on the right corner of the screen. The example result is shown in Figure 6.

$$
d=\frac{d_{r e f} \times p_{r e f}}{p}
$$

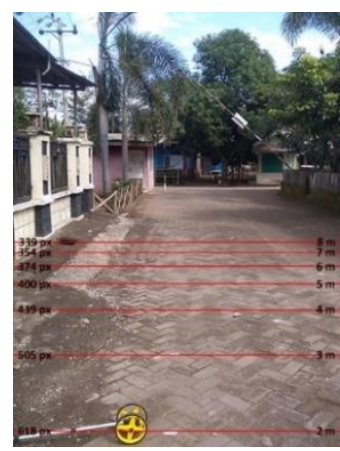

Figure 5. Actual distance and pixel distance comparison

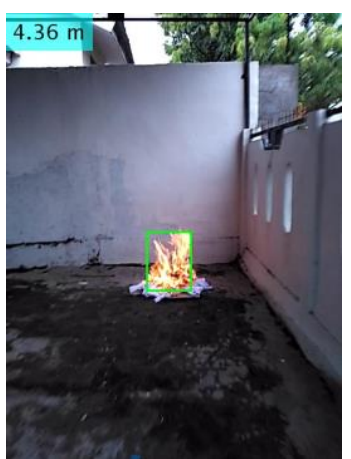

Figure 6. Output of the system

\section{RESULTS AND DISCUSSION}

\subsection{Fire detection}

The results of the fire detection system are expressed in percentage value of accuracy as shown in Figure 7. Video numbers of 1 through 21 are positive data, and the last 14 videos are negative data with 205 frames on average, each video. The system yielded $88.86 \%$ average accuracy. Based on this value, the system can be considered reliable. However, the system performed poorly in some data scenarios due to some factors. For example, in video 33, the system produced only $51.77 \%$ accuracy. In this scenario, the system observed an accelerating vehicle at night time, in which the headlamp had similar color features to fire and appeared to be enlarged continuously. Hence, it exceeded the threshold, and the object was detected as a fire. The example of this case can be seen in Figure 8. The real implementation of this technology has been implemented in one of the blind foundation with 6 respondents from the foundation. The reflection of the fire must also be taken into consideration. The reflection can be detected as fire due to its color, therefore confused about the system. This circumstance only occurred when the system was being used in dark or dim places, which example, as shown in Figure 9. 


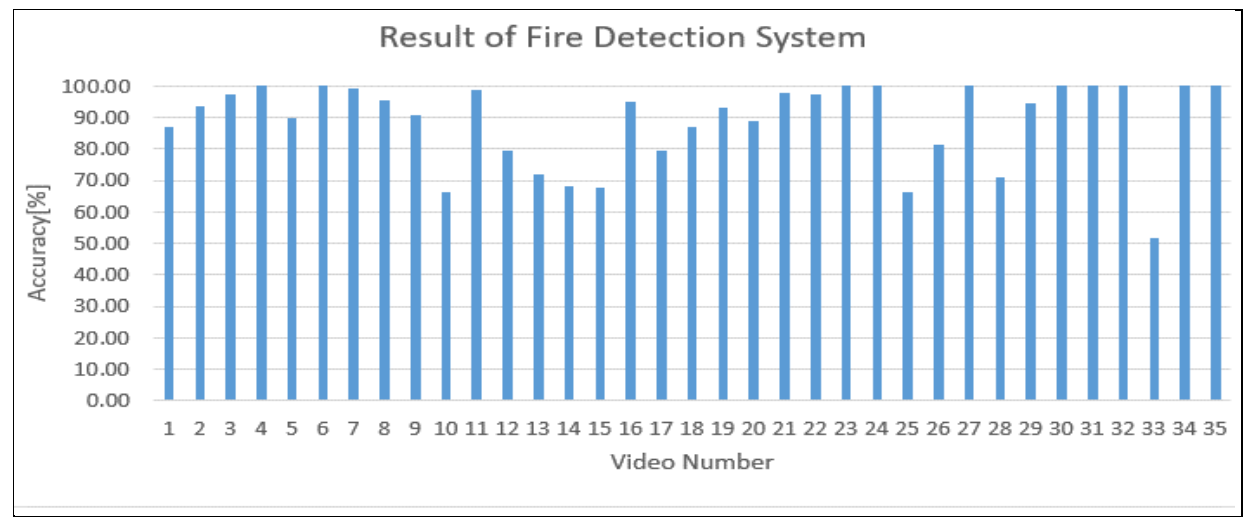

Figure 7. Result of the fire detection system

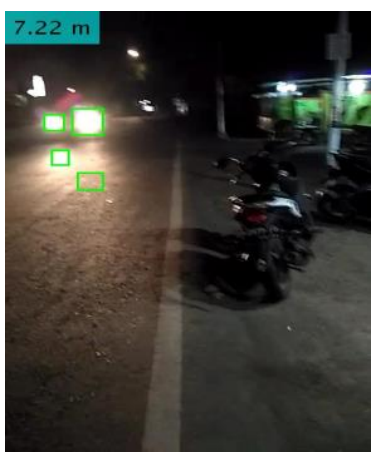

Figure 8. Vehicle headlamp detected as fire

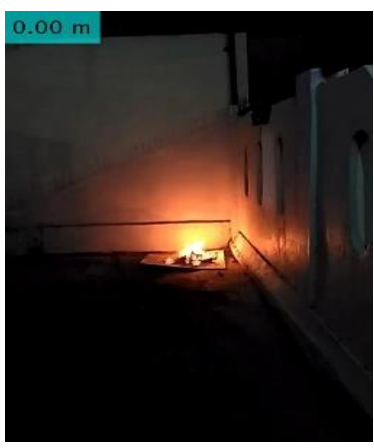

Figure 9. System inability to detect fire that produced a reflection

\subsection{Distance estimation}

The result for the distance estimation system using the pinhole model is validated by calculating its mean square error (MSE). The result can be seen in Table 2. Based on real data, the average time needed by a blind person to reach a distance of 4 meters is 12.22 seconds. System validation is limited to a maximum distance of 4 meters. Because the camera captures 30 frames per second, and the system detects objects every ten frames. Hence, the system can detect objects every 0.33 seconds. Therefore, it can be concluded that it is safe enough for the blind with an average speed of 12 seconds to avoid an obstacle 4 meters ahead.

Table 2. Result of the distance estimation

\begin{tabular}{ccc}
\hline Actual distance $(\mathrm{m})$ & Estimated distance $(\mathrm{m})$ & Square error \\
\hline 2.00 & 2.00 & 0.0000 \\
2.50 & 2.20 & 0.0900 \\
3.00 & 2.99 & 0.0001 \\
3.50 & 3.73 & 0.0529 \\
4.00 & 4.19 & 0.0361 \\
& & 0.0358 \\
\hline
\end{tabular}

The highest error is obtained when the object is 2.5 meters far, where the system estimated it as 2.2 meters. The lowest error is 0 , which occurred at a distance of 2 meters where the actual distance is 2 meters, and the estimated distance is 2 meters. The error occurred because there is a difference in camera angle between the system implementation and the data collection process. The difference in angle causes the distance of the system estimation to differ from the actual distance. Another factor that increases the error value is the height of the camera during system usage. If the height of the camera when the system was being used differs significantly from the height when taking distance data, the scale value used to calculate the distance will also differ, which increases the difference in actual distance and estimated distance. 


\section{CONCLUSION}

In this research, the color model and blob analysis were applied to detect fire, and then the pinhole model is used to estimate fire distance in dynamic scenarios designed for the visually impaired person. The input of the system is 35 videos with a resolution of 480x640 pixels, where 21 videos contained actual footage of fire, and the rest showed objects that have a similar color to fire. The fire detection system using blob analysis yielded $88.86 \%$ accuracy, while the distance estimation using the pinhole model performs well with an MSE value of 0.0358. In the future, the system can be modified to suit broader scopes, such as pothole detection and electric pole detection.

\section{ACKNOWLEDGEMENTS}

This work is supported by the LPPM-Universitas Hasanuddin (UNHAS) via PDUPT-Ristekdikti Grant 2019, Indonesia and AIMP Research Group.

\section{REFERENCES}

[1] FEMA, "Fire Risks for the Blind or Visually Impaired,"Fire Risks Series, 1999.

[2] A. Anwar and S. Aljahdali, "A smart stick for assisting blind people," IOSR Journal of Computer Engineering, vol. 19, no. 3, pp. 86-90, 2017.

[3] S. Adhe, S. Kunthewad, P. Shinde and V. S. Kulkarni, "Ultrasonic smart stick for visually impaired people," IOSR Journal of Electronics and Communication Engineering, vol. 1, pp. 11-15, 2015.

[4] E. G. Dada, A. I. Shani and A. L. Adekunle, "Smart Walking Stick for Visually Impaired People using Ultrasonic Sensor and Arduino," International Journal of Engineering and Technology, vol. 9, no. 5, pp. 3435-3447, 2017.

[5] I. A. Satam, M. N.A. Al-Hamadani and A. H. Ahmed, "Design and Implement Smart Blind Stick," Journal of Advanced Research in Dynamical and Control Systems, vol. 11, no. 8, pp. 42-47, 2019.

[6] S. P. Ramalingam, R. K. Nadesh, and N C Senthilkumar, "Robust face recognition using Enhanced Local Binary Pattern," Bulletin of Electrical Engineering and Informatics, vol. 7, no. 1, pp. 96-101, 2018.

[7] S. J. elias, et al., "Face recognition attendance system using Local Binary Pattern (LBP)," Bulletin of Electrical Engineering and Informatics, vol. 8, no. 1, pp. 239-245, 2019.

[8] R. A. R. Ahmad, M. I. Ahmad, M. N. Md Isa and S. A. Anwar, "Face recognition using assemble of low frequency of DCT feature," Bulletin of Electrical Engineering and Informatics, vol. 8, no. 2, pp. 541-550, 2019.

[9] F. Deeba, H. Memon, F. A. Dharejo. A. Ahmed and A. Ghaffar "LBPH-based enhanced Real-Time Face Recognition," International Journal of Advamced Computer Science and Application, vol. 10, no. 5, pp. 274-280, 2019.

[10] Chunyu Yu, Zhibin Mei and Xi Zhang, "A Real-time Video Fire Flame and Smoke Detection Algorithm," in Procedia Eng., vol. 62, pp. 891-898, 2013.

[11] V. Jaiswal, V. Sharma, S. Varma, "An Implementation of Novel Genetic Based Clustering Algorithm for Color Image Segmentation," Telecommunication, Computing, Electronics and Control, vol. 17, no. 3, pp. 1461-1467, 2019.

[12] Mrunal Pathak, N. Srinivasu and Vinayak Bairagi, "Effective Segmentation of Sclera, Iris And Pupil in Noisy Eye Images," Telecommunication, Computing, Electronics and Control, vol. 17, no. 5, pp. 2346-2354, Oct 2019.

[13] Megistu. Abrham Debasu, "The Effects of Segmentation Techniques in Digital Image Based Identification of Ethiopian Coffee Variety," Telecommunication, Computing, Electronics and Control, vol. 16, no. 2, pp. 713-717, 2018.

[14] G. Yadav, V. Gupta, V. Gaur and M. Bhattacharya., "Optimized flame detection using image processing based techniques," Indian Journal of Computer Science Engineering, vol. 3, no. 2, pp. 202-211, 2012.

[15] Z. Sadreddini, T. Çavdar and H. B. Jond, "A distance measurement method using single camera for indoor environments," 2016 39th International Conference on Telecommunications and Signal Processing (TSP), Vienna, pp. 462-465, 2016.

[16] A. Rahman and N. S. Widodo, "Distance estimation of colored objects in image," 2016 Conference on Fundamental and Applied Science for Advanced Technology (CONFAST 2016), , vol. 1746, 2016.

[17] A. Rahman, A. Salam, M. Islam and P. Sarker, "An image based approach to compute object distance," International Journal of Computational Intelligent System, vol. 1, no. 4, pp. 304-312, 2008.

[18] A. Abdurrasyid, I. Indrianto and R. Arianto, "Detection of Immovable Objects on Visually Impaired People Walking Aids," Telecommunication, Computing, Electronics and Control, vol. 17, no. 2, pp. 580-585, 2019.

[19] P. B. Prakoso and Y. Sari, "Vehicle Detection using Background Subtraction and Clustering Algorithms," Telecommunication, Computing, Electronics and Control, vol. 17, no. 3, pp. 1393-1398, 2019.

[20] H. N. Abdullah and N. H. Abdulghafoor, "Objects Detection and Tracking using Fast Principle Component Purist and Kalman Filter," International Journal of Electrical and Computer Engineering, vol. 10, no. 2, pp. 1317-1326, 2020.

[21] M. A. Soeleman et al., "Adaptive Threshold for Moving Objects Detection using Gaussian Mixture Model," Telecommunication, Computing, Electronics and Control, vol. 18, no. 2, pp. 1122-1129, 2020.

[22] K. Lakhwani, P. D. Murarka and N. S. Chauhan "Color Space Transformation for Visual Enhancement of noisy color Image," International Journal of ICT and Management, vol. 3, no. 2, pp. 9-13, 2015.

[23] Basri, Indrabayu and A. Achmad, "Gaussian Mixture Models optimization for counting the numbers of vehicle by adjusting the Region of Interest under heavy traffic condition," 2015 International Seminar on Intelligent Technology and Its Applications (ISITIA), Surabaya, pp. 245-250, 2015. 
[24] Indrabayu, R. Y. Bakti and I. S. Areni, "A modified pinhole camera model for automatic speed detection of diagonally moving vehicle," Journal of Engineering Science and Technology, vol. 13, no. 6, pp. 1722-1734, 2018.

[25] J. Phattaralerphong, H. Sinoquet, "A method for 3D reconstruction of tree crown volume from photographs: assessment with 3D-digitized plants," Tree Physiology, vol. 25, pp. 1229-1242, 2005.

\section{BIOGRAPHIES OF AUTHORS}

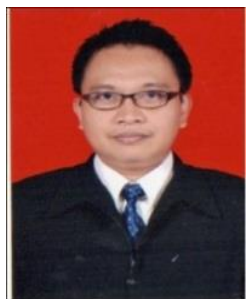

Indrabayu was born on July 16, 1975 in Makassar, Indonesia. He was awarded Summa Cum Laude from the doctoral degree in artificial engineering in civil application from Hasanuddin University, Makassar, Indonesia, in 2013. Also received M.E degree in multimedia and communication from Institut Teknologi 10 November, Surabaya, Indonesia in 2005. His research interest includes artificial intelligence and multimedia processing. Founder and Head of Artificial Intelligence and Multimedia Processing (AIMP) Research Group. Currently work also as secretary of informatics department of Hasanuddin University.

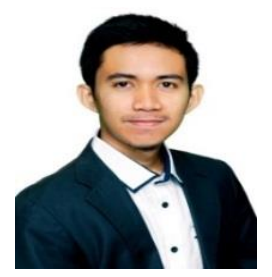

Rahmat Hardian Putra was born in July 1995 in Palopo, South Sulawesi, Indonesia. Graduated elementary to high school in Palopo, then pursuing a bachelor's degree in Makassar, the capital of South Sulawesi. During college, joining Artificial Intelligence and Multimedia Processing (AIMP) and graduated with Cum laude in informatics engineering from Hasanuddin University in 2017. At the end of 2017 joining Telkom Indonesia, the biggest telecommunication company in Indonesia, up until now working on knowledge management in PT. Telkom Indonesia.

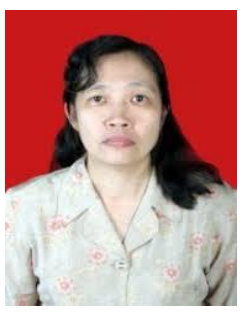

Ingrid Nurtanio received the bachelor degree in electrical engineering from Hasanuddin University, Makassar, Indonesia in 1986. She received her master of technology from Hasanuddin University, Makassar, Indonesia in 2002. She received her doctoral degree from Institut Teknologi Sepuluh Nopember, Surabaya, Indonesia in 2013. Her research interest are digital image processing, computer vision and intelligent system. currently, she is the staff of Department of Informatics, Faculty of Engineering, Hasanuddin University. She is a member of IAENG and IEEE.

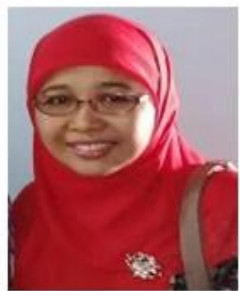

Intan Sari Areni was born in Watampone, South-Sulawesi, Indonesia, 1975. She received a B.E. and M.E degree in electrical engineering from University of Hasanuddin (UNHAS) Makassar (1999) and University of Gadjah Mada (UGM) Jogjakarta (2002), respectively, and received a Doctorate degree from Ehime University Japan in 2013. Since 2000 she has been in the Electrical Engineering Department, Faculty of Engineering UNHAS as a lecturer. Her research interests in multimedia signal processing, telecommunication, wireless and biomedical engineering, powerline communication system (PLC). She is a member of IEEE and IAENG.

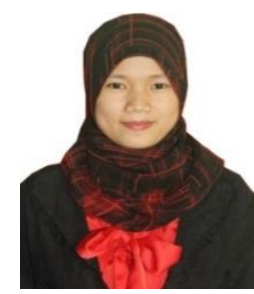

Anugrayani Bustamin was born in Sengkang, 1 December 1990. Graduated S1 Informatics Engineering majors on Universitas Hasanuddin, Makassar. In 2014 continuing education in Informatics Engineering Study Program, Department of Electrical Engineering, Universitas Hasanuddin, Makassar, Indonesia. Member of Artificial Intelligence and Multimedia Processing (AIMP) Research Group, Universitas Hasanuddin. Her research interests include speech recognition and natural language processing. 